\title{
17a-Estradiol is generated locally in the male rat brain and can regulate GAD65 expression and anxiety.
}

\author{
Abbreviated title: $17 \alpha$-estradiol generated in the brain
}

\author{
Takamitsu Ikeda, $\mathrm{PhD}^{1}$, Yukiko Makino, $\mathrm{PhD}^{2}$, Maki K. Yamada, $\mathrm{PhD}^{1,3}$ \\ ${ }^{1}$ Laboratory of Chemical Pharmacology, ${ }^{2}$ Laboratory of Chemistry and Biology, Graduate School of \\ Pharmaceutical Sciences, The University of Tokyo, 7-3-1 Hongo, Bunkyo-ku, Tokyo 113-0033, ${ }^{3}$ PRESTO, \\ The Japan Science and Technology Agency, 4-1-8 Honcho Kawaguchi, Saitama, 332-0012, Japan
}

Number of pages 18; Number of figures 4, tables 0, multimedia and 3D models 0; Number of words for Abstract 228, Introduction 380, Materials and Method 1351, Discussion 1022

Corresponding author address: Maki K. Yamada, PhD, E-mail: makiky-tky@umin.ac.jp, Present address: Physiological Chemistry and Metabolism, Graduate School of Medicine, The University of Tokyo, 7-3-1 Hongo Bunkyo-ku Tokyo Japan 113-0033, Phone +81-3-5841-3496, Fax +81- 3-5841-3603 


\begin{abstract}
Increasing evidence suggests that 176-estradiol, a sex hormone, is synthesized by neurons. In addition, $17 a^{-e s t r a d i o l, ~ t h e ~ s t e r e o i s o m e r ~ o f ~} 176$-estradiol, is reported to be the dominant form in the male mouse brain. However, probably because the method to detect these isomers requires unusual and precise experimental design, the presence of this endogenous $17 \alpha^{-}$estradiol has not been reported subsequently and the actual role is therefore not well elucidated. We first quantified the estradiol level in hippocampal extracts using gas chromatography/mass spectrometry. As a result, 17a-estradiol was found in all of the male rats tested, while that of 176-estradiol was detected only in a certain subset. The estrogen-biosynthesis inhibitor letrozole decreased the expression of the major presynaptic GABA synthesizing enzyme GAD65 in cultured neurons and the effect was abrogated by exogenously supplied 17a-estradiol. Next, injection of the inhibitor into the brain reduced the $17 \alpha$-estradiol level, indicating its biogenesis in the brain. Under the same conditions, immuno-staining of GAD65 was also decreased. Furthermore, the inhibitor treatment increased anxiety index of rats in the open field and this was ameliorated by the addition of $17 \alpha^{-}$estradiol. We showed that $17 \alpha^{-}$-estradiol was generated in thebbrain and acted as a regulator of inhibitory neurotransmission as well as behavior. These results may have implications for a variety of diseases, such as the menopausal depression and Alzheimer's disease that have been reported to be related to estrogen levels.
\end{abstract}

Keywords: Estrogen, female hormone, inhibition, anti-anxiety, aromatase, AKR1C 


\section{Introduction}

While the small chemical compound $17 \beta$-estradiol is known as a female hormone, increasing evidence has demonstrated that it is synthesized by neurons in the brain and functions locally as a neuromodulator (Fester et al., 2011; Srivastava et al., 2013).

In $2005,17 \alpha$-estradiol, the stereoisomer of $17 \beta$-estradiol, was reported to be the dominant form made in the male mouse brain (Toran-Allerand et al., 2005). This stereoisomer differs in the orientation of the $17^{\text {th }}-\mathrm{OH}$ of the steroid structure, and is mainly made from $17 \alpha$-testosterone, while $17 \beta$-estradiol is generated from $17 \beta$-testosterone, both by an enzyme called aromatase. However, the presence of the endogenous $17 \alpha$-estradiol has not been reported subsequently. This is at least partly because the usual quantification methods for $17 " \beta$ "-estradiol have been immunoassays (RIA, ELISA) that are not specific enough. Accordingly, the role of the endogenous $17 \alpha$-estradiol has not been elucidated yet.

It is reported in humans that $17 \alpha$-testosterone, the precursor for $17 \alpha$-estradiol, is measurable in the serum of $24 \%$ of boys and $33 \%$ of girls (Courant et al., 2010). Thus, individual variation in the $17 \alpha$-estradiol concentration is likely. If so, this should be taken into account when considering the therapeutic use of estradiol-related drugs.

We at first detected $17 \alpha$-estradiol in the male rat brain using gas chromatography/mass spectrometry (GC/MS). Then, since an effect of estradiol on GAD65, a dominant GABA synthetic enzyme in the presynaptic terminals of inhibitory neurons (Ikeda et al., 2006; Nakamura et al., 2004; Rudick and Woolley, 2001) as well as direct transcriptional activation (Hudgens et al., 2009) have been reported, we used GAD65 to evaluate the effect of $17 \alpha$-estradiol in comparison with $17 \beta$-estradiol. Next, we sought 
to inhibit estrogen synthesis in the brain by intracerebroventricular injection of an inhibitor to confirm the biogenesis and role in the male rat brain. We thereby found the first evidence of locally-generated $17 \alpha$-estradiol and its effects on the GAD level in vitro and in vivo, as well as effect on anxiety-related behavior.

Since there have been many reports on the different functions of the exogenously applied 17 $\alpha$-estradiol and 17 $\beta$-estradiol (Barha and Galea, 2010), it seems plausible that this difference could be utilized to confer benefit in certain cases.

The present results provide a basis for further analyses on the variety exhibited in different individuals and the specific functions of $17 \alpha$-estradiol.

\section{Materials and Methods}

Animals All experiments were performed with Wistar/ST rats from SLC (Shizuoka, Japan), according to the Japan Neuroscience Society guide for the care and use of laboratory animals under the approval of the Committee for Animal Experiments of the University of Tokyo.

Quantification of the estradiol level using GC/MS Cold phosphate-buffered saline was flushed via the cardiac ventricles to purge blood of ether-anesthetized male rats, then the hippocampi were rapidly dissected on ice and homogenized by a teflon homogenizer at $1000 \mathrm{rpm} 10$ times in 3 volumes of chilled water. The known amounts of isotopes, $17 \alpha$-estradiol-2,4- $d_{2}$ and $17 \beta$-estradiol-2,4,16,16,17- $d_{5}$ (CDN isotope Quebec, Canada) were added to the homogenate, so that they served as the internal standards for the extraction, derivatization and all other procedures. They can be 
detected at the final quantification using GC/MS as molecules having the same retention time but different masses. Ethylacetate (16 vol.) was added to the homogenate, mixed by a vortex mixer for 10 minutes and centrifuged $2000 \times \mathrm{g}$ at $10^{\circ} \mathrm{C}$ for 10 minutes. The upper organic layer was collected and the solvent was evaporated under a stream of nitrogen gas at room temperature. $100 \mu 1$ of 10\%TMCS in BSTFA (Pierce) and $60 \mu 1$ of pyridine (Sigma-Aldrich) were added to the residue, and heated at $70^{\circ} \mathrm{C}$ for $60 \mathrm{~min}$ to prepare the derivative. The solvent was evaporated and residue was resolved in chloroform.

The Ultra-2 capillary column ( $25 \mathrm{~m}$ long, $0.25 \mathrm{~mm}$ in diameter, $0.33 \mu \mathrm{m}$ film coated; Agilent Technologies, Santa Rosa, CA) using Herium as the carrier gas was used for high-resolution selected ion monitoring mode of GC/MS. (6890N GC, Agilent technologies Santa Rosa, CA, USA, coupled with JMS-SX102A MS, JEOL, Tokyo, Japan). The injection port and transfer line temperature were maintained at $250^{\circ} \mathrm{C}$ and $300^{\circ} \mathrm{C}$, respectively. The program of the $\mathrm{GC}$ oven temperature was as follows: $70^{\circ} \mathrm{C}$ for $5 \mathrm{~min}$, followed by a ramp up to $160^{\circ} \mathrm{C}$ at $30^{\circ} \mathrm{C} / \mathrm{min}$, then to $300^{\circ} \mathrm{C}$ at $7^{\circ} \mathrm{C} / \mathrm{min}$, and a final hold for $7 \mathrm{~min}$. The presence of estradiol was confirmed by the mass of $\mathrm{m} / \mathrm{z}$ 416.2567 and that of the structurally expected fragments $(\mathrm{m} / \mathrm{z} 326.2066$ and $\mathrm{m} / \mathrm{z}$ 285.1675), in addition to having the same retention time with both pure standards and mixed isotopes. We usually load the pure estradiols as external standards just before every set of experiments, and could detect $0.3 \mathrm{pg}$ of the standards in charts, so the detection limit of GC/MS is thought to be comparable to or better than the radioimmuno-assay system. 
Primary Neuronal Culture Hippocampal neurons were cultured by a previously described method (Ohba et al., 2005), except using Neurobasal medium free of phenol red (Invitrogen, San Diego, CA) as a possible estrogen-like molecule. In brief, dispersed neurons from the hippocampi (both male and female rats on embryonic day 18) were suspended in medium containing 10\% fetal bovine serum (MP Biomedicals Inc., Irvine, CA) and were plated at a density of 50,000 cells $/ \mathrm{cm}^{2}$ on polyethyleneimine (Sigma, St. Louis, MO)-coated glass coverslips (Matsunami Glass Osaka, Japan) attached to the flexiPERM having eight wells of $0.7 \times 1.0 \mathrm{~cm}^{2}$ each (Sartorius, Göttingen, Germany). Medium was replaced with a serum-free one supplemented with 2\% B27 (Invitrogen) $24 \mathrm{hr}$ after plating. Half of the medium was gently changed every other day. The aromatase inhibitor letrozole (kindly provided by Novartis Pharma AG, Basel, Switzerland), with or without $17 \alpha$ - or $17 \beta$ - estradiol (Sigma-Aldrich, St. Louis, MO), was added to the new medium at the change on 7 days of in vitro and applied for $48 \mathrm{hr}$.

Intracerebroventricular injection of letrozole Each Wistar/ST male rat (SLC, 4 wk-old) was anesthetized with pentobarbital, and a guide cannula with an inserted plug was intracerebrally implanted into the lateral cerebral ventricle, one week before the injection. The implantation site was adjusted by the bregma-lambda length to a place corresponding to $0.8 \mathrm{~mm}$ anteroposterior, $1.4 \mathrm{~mm}$ lateral right and $3 \mathrm{~mm}$ dorsoventral in the rat atlas (Paxinos and Watson, Academic Press). A solder-mounted wire ring on the cannula was fixed to the skull with a screw and dental cement. For injection, the plug was transiently removed and vehicle (20\% DMSO / 80\% saline) or letrozole $(1.4 \mu \mathrm{g} / \mathrm{kg})$ was injected every 24 hours for three days, then samples were taken 24 hours after the last injection. For the behavior test, to avoid stress from the daily handling, a tube from 
the subcutaneously-implanted osmotic pump (Alzet, Cupertine, CA, USA) was connected to the injection needle in the guide cannula for three days. The dose of letrozole $(1.4 \mu \mathrm{g} / \mathrm{kg} / \mathrm{day})$ with or without $17 \alpha$-estradiol (30 $\mu \mathrm{g} / \mathrm{kg} / \mathrm{day})$ was determined according to previous reports (Liang et al., 2002).

Immunostaining and Fluorescence Image Analysis The cultured cells were treated sequentially with $4 \%$ paraformaldehyde at $4{ }^{\circ} \mathrm{C}$ for $30 \mathrm{~min}$, wash, $0.1 \%$ Triton $\mathrm{X}-100$ for $15 \mathrm{~min}$, wash and $1 \%$ goat serum/PBS for $1 \mathrm{hr}$, and then with primary antibody for GAD65 (GAD-6, mouse monoclonal, $6 \mu \mathrm{g} / \mathrm{ml}$, DSHB) overnight. The specificity of the GAD-6 was previously characterized as a single band at the size of GAD65 on Western analysis, in a literature (Chang and Gottlieb, 1988) and by ourselves. After washing, the cells were incubated with a secondary antibody (Alexa594-conjugated anti-mouse IgG; dilution, 1:500; Invitrogen) for $2 \mathrm{hr}$ at $4^{\circ} \mathrm{C}$.

For immunohistochemistry, rats were anesthetized with sodium pentobarbital (50 $\mathrm{mg} / \mathrm{kg}$, i.p.) and transcardially perfused with chilled phosphate-buffered saline followed by $4 \%$ paraformaldehyde in $0.1 \mathrm{M}$ phosphate buffer $(\mathrm{PB}, \mathrm{pH} 7.4)$. The brains were post-fixed $\left(4^{\circ} \mathrm{C}, 1.5 \mathrm{~h}\right)$, immersed in $10 \%, 20 \%$, and $30 \%$ sucrose in $\mathrm{PB}$ in series $\left(4^{\circ} \mathrm{C}\right.$, $24 \mathrm{hr}$ in total), frozen and coronally sectioned at a thickness of $30 \mu \mathrm{m}$. Sections mounted on glass slides were incubated in $0.1 \%$ Triton $\mathrm{X}-100$ for $10 \mathrm{~min}, 5 \%$ goat serum for $1 \mathrm{hr}$ at $4{ }^{\circ} \mathrm{C}$, GAD-6 antibody in $0.25 \%$ goat serum at $4^{\circ} \mathrm{C}$ overnight, and then with the Alexa594 labeled antibody in $1 \%$ goat serum with the addition of NeuroTrace $435 / 455$ (fluorescent Nissl, Invitrogen) for $1 \mathrm{hr}$ at room temperature.

All 8-bit images were captured with a Orca-II cooled-CCD camera (Hamamatsu photonics, Shizuoka, Japan) on a Nikon TE300 microscope $(10 \times$ objective lens $)$. Care 
was taken not to include intensity-saturated pixels. Compared images were taken in the same conditions.

For neuronal culture, the image analysis method had been developed in the previous study (Ikeda et al., 2006). Briefly, 10 image frames $\left(340 \times 340 \mu \mathrm{m}^{2}\right)$ were randomly selected from a well according to marks on the glass bottom assigned in a blind manner. To omit background staining, the average of mode $+2 \times$ standard deviation of the intensity value in the images from control wells (cells with no reagent) of each batch of culture was calculated and defined as a threshold value. This nearly matches the intensity value from the glass surface without any evident neurons, possibly from antibodies remaining on the coated glass. Values greater than the threshold were integrated for each image and averaged, normalized to control. The intensity was measured by Scion Image software (Scion Corp., Frederick, MD).

For the slice imaging, the focus points and image frames were determined using filter-set for Nissl staining then changed to that for Alexa594 to preserve fluorescence and to keep unbiased selection. The sub-region was also objectively defined by Nissl images taken together and the raw intensity was used for quantification. The thresholding procedure was not done because of the apparently low backgrouond. The analysis was done by Image $(\mathrm{NIH})$ and MetaMorph (Universal Imaging Corp., West Chester, PA).

Behavior test The male rats were received daily handling before the surgery, until they seemed to be habituated. For the open-field test, each rat was put at the center of an unfamiliar, round open-top arena with a wall $(37.5 \mathrm{~cm}$ diameter, 80-100 lux) and a movie was taken from above for $5 \mathrm{~min}$. Each image from the movie was binarized for 
body shape and the center of the mass position was examined to determine whether it came into the center of the arena $(22.5 \mathrm{~cm}$ diameter $)$ or not and used to draw the track in the Fig. 4A.

Statistical analysis Group data are presented as mean \pm SEM. Welch's modified $t$-test for comparison of two groups or Tukey's test for multiple comparisons after ANOVA was applied by Microsoft Excel or R, respectively.

\section{Results}

\section{Quantification of estradiol in the male rat hippocampus}

For the initial step, we quantified the $17 \alpha$ - and $17 \beta$ - estradiol present in the hippocampus utilizing their different retention times in GC due to the large conformational difference between these stereoisomers. When preparing the samples, we flushed the blood out and added an internal control for quantification. As seen in the quantitative data (Fig. 1A-C), we detected $17 \alpha$-estradiol in all of the samples, but only three out of five rats exhibited a detectable amount of $17 \beta$-estradiol. Of the 17 rats tested, including those in Fig. 3A, we found a detectable level of $17 \alpha$-estradiol in all of them, while $17 \beta$-estradiol was detected in only a portion.

\section{The effect of $17 \alpha-$ and $17 \beta$ - estradiol on cultured hippocampal neurons}

$17 \alpha$-Estradiol has been described as "inactive" in certain previous studies (Lingham et al., 1988; Womble et al., 2002). Therefore, to clearly show the effect of $17 \alpha$-estradiol, we added $17 \alpha$ - and $17 \beta$-estradiol to the medium of a relatively pure neuronal population 
together with an inhibitor of aromatase, an enzyme required for estrogen biogenesis. After confirming a decrease in the GAD65 signal induced by letrozole, as in the previous findings (Ikeda et al., 2006), the dose for recovery was studied. As the result, $17 \alpha$-estradiol was found to be comparable to or more effective than $17 \beta$-estradiol in terms of the GAD65 signal levels (Fig. 2, ${ }^{* *} P=0.0084$ vs vehicle control, ${ }^{\# \#} P<0.01$ vs the letrozole-only treated cultures, $P>0.05$ for all pairs of the same dose of $17 \alpha$ - and 17ß-estradiol, $\mathrm{n}=7-25$ wells).

\section{Decrease of estradiol by a brain injection of an aromatase inhibitor}

To further inhibit the biogenesis of estradiol in the brain separately from other tissues, such as the adrenal glands and adipose tissue, we injected letrozole directly into the brain ventricles. Since the brain is separated from other tissues by the blood-brain barrier, this route of administration limits the effectiveness of the drug in the brain. As a result, a significant decrease in the $17 \alpha$-estradiol level was found in the letrozole-treated rats (Fig. 3A, ${ }^{* * P} P=0.0097 . \mathrm{N}=5$ and 7 rats).

\section{An aromatase inhibitor down-regulated the brain GAD65 level}

After 3-days of letrozole injection i.c.v., immunostaining by anti-GAD65 antibody (specificity: a single band on Western analysis) detected a decrease in the immunoreactive signals (Fig. 4A, B). In the vehicle control sections, a potent GAD65 signal was seen around the pyramidal cell layer (stratum pyramidale, SP) and a weak signal in the stratum oriens (SO) and stratum radiatum (SR). By semiquantification of the GAD65-signal intensity, a significantly lowered signal was found in the letrozole-treated rats in the pyramidal cell layer (Fig. $4 \mathrm{C}, 85 \pm 5 \%, * P=0.038$ ). The 
difference was not evident in CA1-SO $(97 \pm 3 \%, P=0.51)$ containing basal dendrites, or CA1-SR $(92 \pm 2 \%, P=0.05)$ containing apical dendrites. It was, however, observed in CA3a (which is closest to CA1 among CA3 subregions a, b, c, $68 \pm 6 \%$ in the SO and $75 \pm 4 \%$ in the SP, $P s<0.05)$ and in the molecular layer of the dentate gyrus $(75 \pm 7 \%$, $P<0.05)$ in the hippocampal formation.

\section{Rat behavior was altered by letrozole treatment and this effect was canceled by the addition of $17 \alpha$-estradiol}

We analyzed anxiety-related behavior in the open field test of the letrozole-treated rats based on the fact that GABA-enhancing drugs are commonly used as anti-anxiety drugs. In this test, a shortened length of time spent in the center is generally considered as an index of the level of anxiety. After 3 days of letrozole injection into cerebroventricles, the time in the center was shorter in the letrozole-treated group and a simultaneous injection of $17 \alpha$-estradiol abrogated the effects of letrozole. (Fig. 5A, B left, $* P=0.032$

vehicle vs letrozole, ${ }^{\#} P=0.047$ letrozole vs letrozole $+17 \alpha \mathrm{E}, \mathrm{N}=12,10,11$ rats.) The traveled distance was not significantly different (Fig. 5A, B right, $P>0.05$ ).

\section{Discussion}

We detected $17 \alpha$-estradiol in the male rat brain. We also demonstrated that aromatase inhibitor injection in male rat cerebral ventricles decreased $17 \alpha$-estradiol levels, indicating that this molecule is generated in the brain, even in males. In addition, aromatase inhibition altered GAD65 expression as well as anxiety-related behavior, which effects were abrogated by the addition of $17 \alpha$-estradiol. These results suggest that 
the $17 \alpha$-estradiol generated in the brain changes mood, possibly via modulation of transmission.

\section{$17 \alpha$-Estradiol is produced in the brain}

It has been proposed that $17 \alpha$-estradiol is produced in the brain since it was detected in the mouse brain and its presence was not affected by ovariectomy or adrenalectomy (Toran-Allerand et al., 2005). Our result of an intracerebroventricular injection of letrozole supports this previous report.

However, a few papers have reported detection by chromatography of brain $17 \beta$-estradiol without $17 \alpha$-estradiol. We consider this discrepancy to be explained by the difference in methods and/or the strain of animal used.

In terms of the method, there are possible advantages as follows; 1, a high-resolution selected ion monitoring mode by GC/MS; 2, high temperature in GC favorable to dissociating disturbing molecular interactions; 3, the detection system of MS that minimizes steps to the detection; 4, quick perfusion and dissection under chilled conditions; 5, homogenization with water so as to osmotically punctuate cellular organelles; 6 , internal standards maintained throughout the sample processing.

Otherwise, differences in the sample source, such as animals being from a different strain/colony, might also lead to a difference in the detected stereoisomer. Even within the single closed-colony strain of rats we used (Wistar/ST), the ratio of $17 \alpha-$ and $17 \beta$-estradiol in each individual varied considerably (Fig.1). This strongly suggests that the generation system can vary even within animals of the same strain. The most probable cause is a genetic variation, such as single nucleotide polymorphisms in the AKR1C family that can act as 17 $\alpha$-hydroxysteroid dehydrogenase that generates the 
precursor $17 \alpha$-testosterone for the aromatase (Faucher et al., 2006). Although it is not yet possible to resolve the cause underlying the observed heterogeneity in stereoisomer expression, future analyses of the variance of this and other related enzymes, such as those in the AKR1C family, should shed light on such inter-individual variations in laboratory animals as well as in humans.

Despite the differences among various reports in the literature, the presence of endogenous $17 \alpha$-estradiol in the brain at least in this strain of rats was clearly confirmed in the present study.

\section{7a-estradiol is a positive regulator of the inhibitory synaptic molecule, GAD65}

We used a neuronal culture system for the detailed recovery experiments (Fig. 2), because this system has the advantage that any effect other than that from neurons is negligible. As a result, clear data were obtained. The similar dose dependency of $17 \alpha-$ to $17 \beta$-estradiol confirmed that the residual contamination of $17 \beta$-estradiol in the purchased $17 \alpha$-estradiol should not be the sole effector. The effective estradiol dose in this experiment $(\sim 10 \mathrm{nM})$ can induce ER $\alpha$ to bind the GAD65 promotor (Hudgens et al., 2009). Thus, of the possible affected factors including the major estradiol metabolite estriol, the estradiols were most probably the direct cause of GAD recovery (Kuiper et al., 1997). The recovery showed that, in the presence of letrozol, neurons were able to respond to exogenous $17-\alpha$ estradiol in their GAD regulation and return to baseline. Thus, it is reasonable to think that the halted biogenesis of endogenous estradiol was the main cause of the observed GAD65 decrease. This experiment clearly demonstrated a $17 \alpha$-estradiol effect on GABAergic function and supports the notion of an endogenous estradiol regulation of GAD65. 


\section{The pattern of GAD65 decrease and its significance}

We first found the effect of letrozole that decreases the GAD65 level in the live brain

(Fig. 3). In addition, the pattern of decrease around the neuronal soma is suggestive, because this perisomatic inhibition is thought to be important in memory processing (Paulsen and Moser, 1998; Royer et al., 2012). That is, the estradiol produced by neurons accelerates perisomatic inhibition along with GAD65 upregulation and thus helps maintain normal memory function. Together with the reported synergistic effect of BDNF on GAD (Ikeda et al., 2006; Ohba et al., 2005), locally-generated estradiol may exert an effect on memory processing via control of the GAD level.

\section{The selectivity of $17 \alpha$-estradiol function in the brain}

Although the present paper showed a similarity between the activity of the two isomers, there have been many reported phenomena that are only induced by $17 \beta$-estradiol, not 17 $\alpha$-estradiol, and vice versa (Barha and Galea, 2010). Nevertheless, the binding constants $(\mathrm{Kd})$ of both of these isomers to the two known receptors, ER $\alpha$ and ER $\beta$, were shown to be the same (Toran-Allerand et al., 2005) or similar (Kuiper et al., 1997). Considering that a ligand with the same affinity for a given receptor theoretically ought to elicit the same response, this apparent discrepancy may be due to either modulators of the known receptors, ligand-specific carrier proteins or unknown additional receptors that might be more selective for one of isomers (Snyder et al., 2010; Toran-Allerand et al., 2005). Considering purpose-driven nature of organisms, an unknown mechanism for the different responses is suggested by the existence of endogenous $17 \alpha$-estradiol.

\section{Possible implications for treatment}


We found an increase in the anxiety-related behavior in the letrozole injected rats, consistent with the many reports on estrogen's effect on relieving anxiety. The present findings add information about the potential role of endogenous $17 \alpha$-estradiol and the temporal association of behavioral changes with decrease in GAD-intensity. Even though a causal relationship is yet to be determined, our results suggest that some estradiol functions may be induced via GAD regulation. Given this possibility, treatment of menopausal women or letrozole-induced depression with GABA-enhancers should perhaps be considered. Furthermore, if $17 \alpha$-estradiol safely works more preferentially in the brain than $17 \beta$-estradiol, many other therapeutic options may also become available.

The differences in these isomers would be clarified by further investigation involving the wide range of information available on the activity of estradiol (Balthazart and Ball, 2006; Daniel, 2013). Applications for estradiol-related drugs for psychiatric and/or neurological disorders should be carefully examined and analyzed based on these differences as well as individual variations. 


\section{Disclosure/Conflict of Interest}

The authors declare no competing financial interests.

\section{Acknowledgements}

This work is supported by Grant-in-Aid from Scientific Research (C, 19590060), Priority Areas Integrative Brain Research (20019016) from the MEXT Japan and PRESTO from JST for MKY.

We thank Novartis for providing letrozole, Developmental Studies Hybridoma Bank (DSHB, University of Iowa) for the monoclonal antibody for GAD65 named as GAD6 and Professor Norio Matsuki for supporting our research. 


\section{References}

Balthazart, J., Ball, G. F., 2006. Is brain estradiol a hormone or a neurotransmitter? Trends Neurosci 29, 241-249.

Barha, C. K., Galea, L. A. M., 2010. Influence of different estrogens on neuroplasticity and cognition in the hippocampus. Biochimica Et Biophysica Acta-General Subjects 1800, 1056-1067.

Chang, Y. C., Gottlieb, D. I., 1988. Characterization of the proteins purified with monoclonal antibodies to glutamic acid decarboxylase. J Neurosci 8, 2123-2130.

Courant, F., Aksglaede, L., Antignac, J. P., Monteau, F., Sorensen, K., Andersson, A. M., Skakkebaek, N. E., Juul, A., Bizec, B. L., 2010. Assessment of circulating sex steroid levels in prepubertal and pubertal boys and girls by a novel ultrasensitive gas chromatography-tandem mass spectrometry method. J Clin Endocrinol Metab 95, 82-92.

Daniel, J. M., 2013. Estrogens, estrogen receptors, and female cognitive aging: the impact of timing. Horm Behav 63, 231-237.

Faucher, F., Pereira de Jesus-Tran, K., Cantin, L., Luu-The, V., Labrie, F., Breton, R., 2006. Crystal structures of mouse 17alpha-hydroxysteroid dehydrogenase (apoenzyme and enzyme- $\mathrm{NADP}(\mathrm{H})$ binary complex): identification of molecular determinants responsible for the unique 17alpha-reductive activity of this enzyme. J Mol Biol 364, 747-763.

Fester, L., Prange-Kiel, J., Jarry, H., Rune, G. M., 2011. Estrogen synthesis in the hippocampus. Cell Tissue Res 345, 285-294.

Hudgens, E. D., Ji, L., Carpenter, C. D., Petersen, S. L., 2009. The gad2 Promoter Is a Transcriptional Target of Estrogen Receptor (ER) alpha and ER beta: A Unifying Hypothesis to Explain Diverse Effects of Estradiol. Journal of Neuroscience 29, 8790-8797.

Ikeda, T., Matsuki, N., Yamada, M. K., 2006. Estrogen produced in cultured hippocampal neurons is a functional regulator of a GABAergic machinery. J.Neurosci.Res. 84, 1771-1777. Kuiper, G. G., Carlsson, B., Grandien, K., Enmark, E., Haggblad, J., Nilsson, S., Gustafsson, J. A., 1997. Comparison of the ligand binding specificity and transcript tissue distribution of estrogen receptors alpha and beta. Endocrinology 138, 863-870.

Liang, Y. Q., Akishita, M., Kim, S., Ako, J., Hashimoto, M., Iijima, K., Ohike, Y., Watanabe, T., Sudoh, N., Toba, K., Yoshizumi, M., Ouchi, Y., 2002. Estrogen receptor beta is involved in the anorectic action of estrogen. Int.J.Obes.Relat Metab Disord. 26, 1103-1109.

Lingham, R. B., Stancel, G. M., Loose-Mitchell, D. S., 1988. Estrogen regulation of epidermal growth factor receptor messenger ribonucleic acid. Mol.Endocrinol. 2, 230-235.

Nakamura, N. H., Rosell, D. R., Akama, K. T., McEwen, B. S., 2004. Estrogen and Yamada 15 
ovariectomy regulate mRNA and protein of glutamic acid decarboxylases and cation-chloride cotransporters in the adult rat hippocampus. Neuroendocrinology 80, 308-323.

Ohba, S., Ikeda, T., Ikegaya, Y., Nishiyama, N., Matsuki, N., Yamada, M. K., 2005. BDNF locally potentiates GABAergic presynaptic machineries: target-selective circuit inhibition. Cereb Cortex 15, 291-298.

Paulsen, O., Moser, E. I., 1998. A model of hippocampal memory encoding and retrieval: GABAergic control of synaptic plasticity. Trends Neurosci 21, 273-278.

Royer, S., Zemelman, B. V., Losonczy, A., Kim, J., Chance, F., Magee, J. C., Buzsaki, G., 2012. Control of timing, rate and bursts of hippocampal place cells by dendritic and somatic inhibition. Nat Neurosci 15, 769-775.

Rudick, C. N., Woolley, C. S., 2001. Estrogen regulates functional inhibition of hippocampal CA1 pyramidal cells in the adult female rat. J Neurosci 21, 6532-6543.

Snyder, M. A., Smejkalova, T., Forlano, P. M., Woolley, C. S., 2010. Multiple ERbeta antisera label in ERbeta knockout and null mouse tissues. J Neurosci Methods 188, 226-234. Srivastava, D. P., Woolfrey, K. M., Penzes, P., 2013. Insights into rapid modulation of neuroplasticity by brain estrogens. Pharmacol Rev 65, 1318-1350.

Toran-Allerand, C. D., Tinnikov, A. A., Singh, R. J., Nethrapalli, I. S., 2005. 17alpha-estradiol: a brain-active estrogen? Endocrinology 146, 3843-3850.

Womble, M. D., Andrew, J. A., Crook, J. J., 2002. 17beta-Estradiol reduces excitatory postsynaptic potential (EPSP) amplitude in rat basolateral amygdala neurons. Neurosci.Lett. 331, 83-86. 


\section{Figure legends}

Figure 1, Simultaneous quantification of the $17 \alpha$ - and $17 \beta$ - estradiols in hippocampal extracts.

(A) Each plot (circle) represents the amount of $17 \alpha$-estradiol (Y-axis) and $17 \beta$-estradiol (X-axis) in one male Wister/ST rat. The data can be grouped with those that exhibited a non-detectable amount of $\beta$-estradiol (group B) and the others (group C).

$(\mathrm{B}, \mathrm{C})$ The representative $\mathrm{GC}$ chart from group $\mathrm{B}(\mathrm{B})$ or $\mathrm{C}(\mathrm{C})$ in Fig1A at the molecular mass of estradiol $(\mathrm{m} / \mathrm{z} 416.2567)$. The $\mathrm{x}$-axis is the retention time and the arrows mark the retention time of the pure estradiol standards, as confirmed by the elution of the isotopes included as internal standards.

Figure 2 , Recovery by $17 \alpha$ - and $17 \beta$-estradiol of the reduced GAD65 level induced by halted estradiol biogenesis in the cultured hippocampal neurons.

The filled triangles show the effect of the aromatase inhibition by letrozole (48 hours, bar under the $\mathrm{X}$-axis), indicating that the neuron-generated estradiol has maintained the GAD65 level. The circles (mean + or - SEM) indicate the effects of the simultaneously added $17 \alpha$ - (open circles) and 17 $\beta$-estradiol (filled circles) at the indicated dose (nM). ** $P<0.01$ vs vehicle control, ${ }^{\# \#} P<0.01$ vs the letrozole-only treated cultures, $\mathrm{n}=7-25$ wells from 5 independent cultures.

Figure 3, Quantification of 17a-estradiol in rat hippocampus after 3-days of injection of Yamada 17 
letrozole, the estradiol biogenesis inhibitor, into brain ventricles.

Quantification of $17 \alpha$-estradiol in the hippocampus. Bars represent the mean + SEM from vehicle (left) or letrozole (right) injected rats. $* * P<0.01, \mathrm{~N}=5,7$ rats.

Figure 4, GAD65 decrease after 3-days of injection of letrozole.

(A) Representative immunohistochemical staining for GAD65, a GABA synthetic enzyme in the hippocampus CA1 in rats after the injection of vehicle (upper) or letrozole (lower). The subregions (stratum pyramidale, SP; stratum oriens, SO; and stratum radiatum, SR) were objectively defined by Nissl staining. Bars $=50 \mu \mathrm{m}$.

(B) Representative images of sections from vehicle- (left) and letrozole- (right) injected rats. The left-most black arrowhead points to a labeled cell body, probably of an inhibitory GABAergic neuron. Other puncta signals are thought to be from presynaptic terminals around CA1 pyramidal somata, which appear as hollow black areas. Bar $=10$ $\mu \mathrm{m}$.

(C) A significant difference in the GAD65-immunostaining intensity was found in the CA1 cell layer, SP. Bars represent the mean + SEM from vehicle (left) or letrozole (right) injected rats. $* P<0.05(\mathrm{~N}=6$ rats each).

Figure 5, Open-field test for the rat anxiety level.

(A) Each streak shows the representative track of a rat in the indicated group in a novel round arena for 5 minutes. (B) The shortened time spent in the center of the field (left Yamada 18 
graph) was used as the increased anxiety index. Data were from vehicle-injected negative control (left), letrozole-injected rats (center) and letrozole plus $17 \alpha$-estradiol (17 $\alpha$-E2) co-injected rats (right). $* P<0.05$ vehicle vs letrozole and ${ }^{\#} P<0.05$ letrozole vs $+17 \alpha$-estradiol $(\mathrm{N}=10-12$ rats). Similar values for traveled distance across the groups (right graph) demonstrated that the differences observed should not be attributed to differences in voluntary locomotor activity. 
Fig. 1
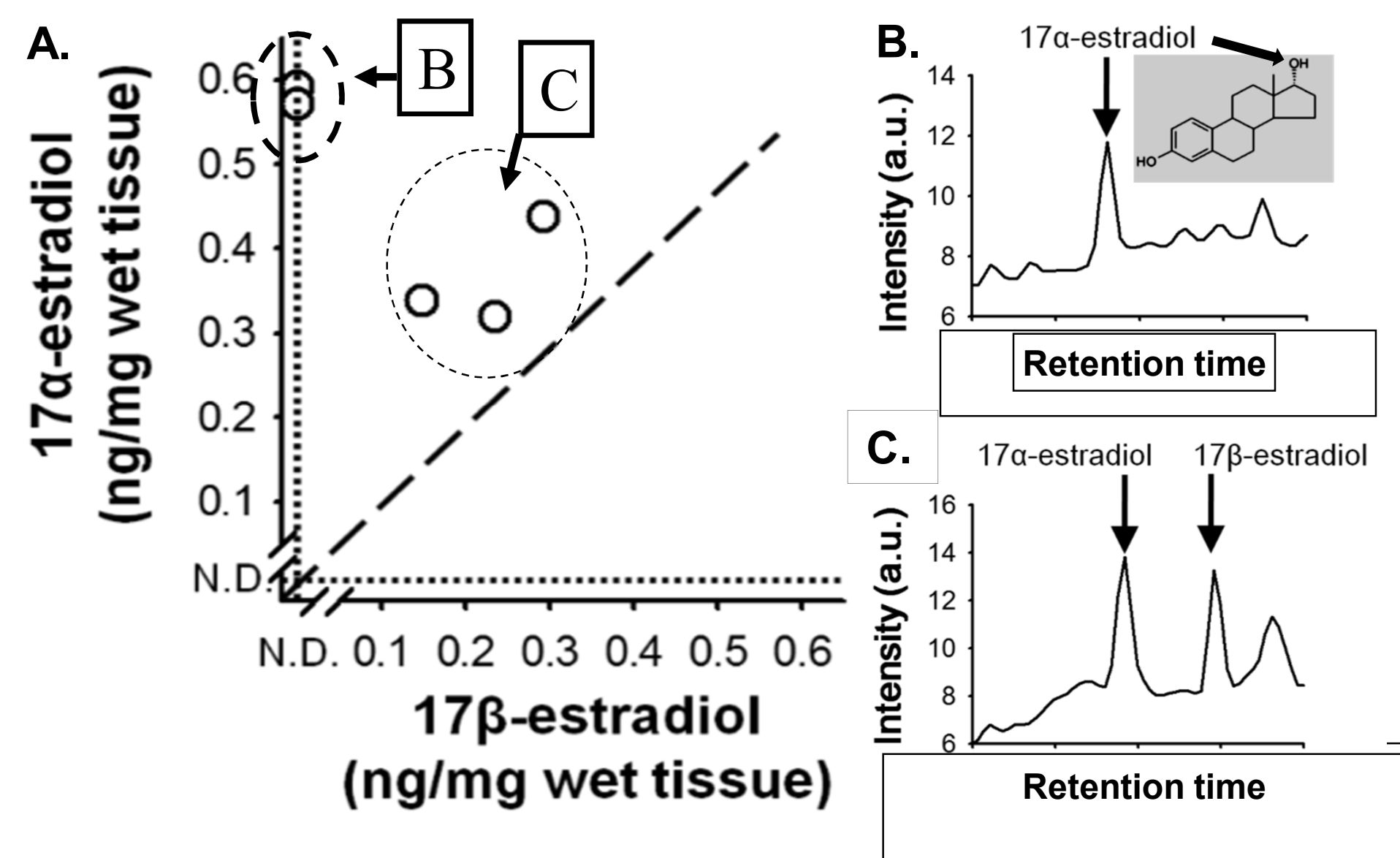
Fig. 2

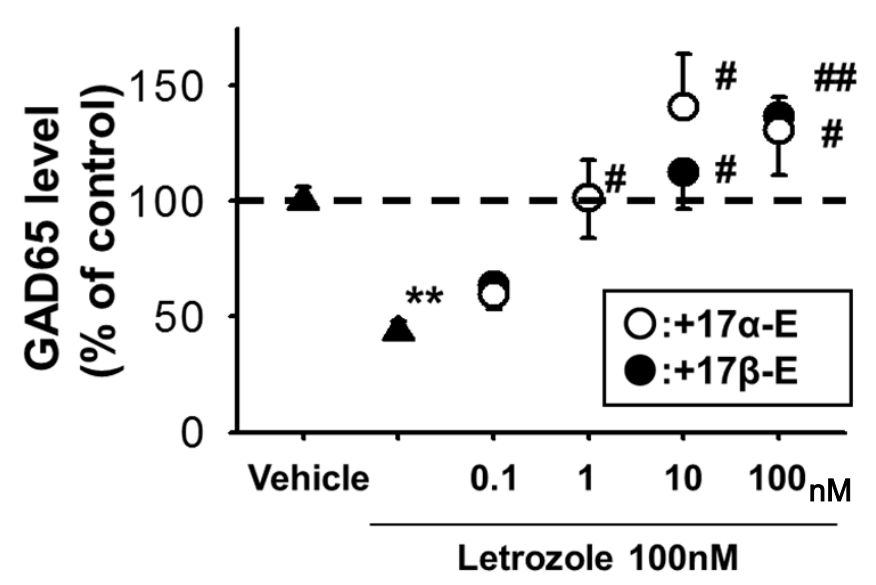


Fig. 3

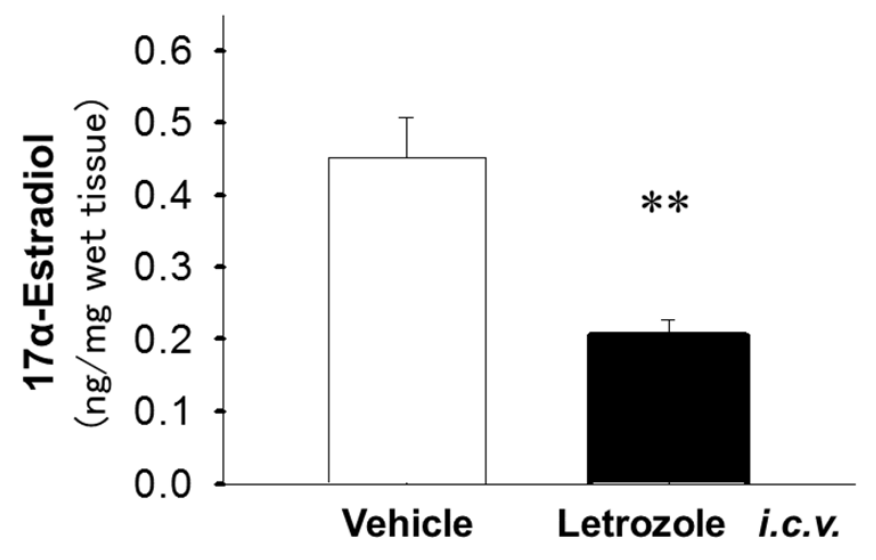


Fig. 4

\section{A GAD65}

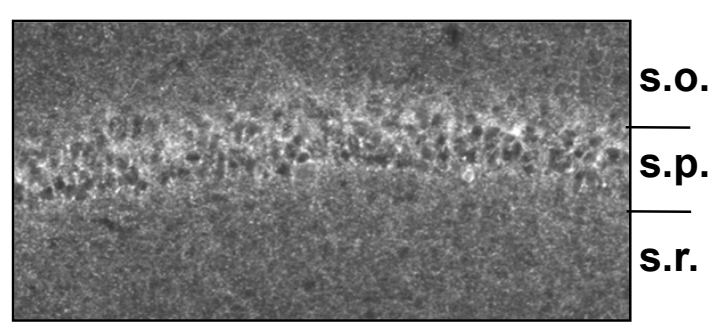

+ Letrozole i. c.V.
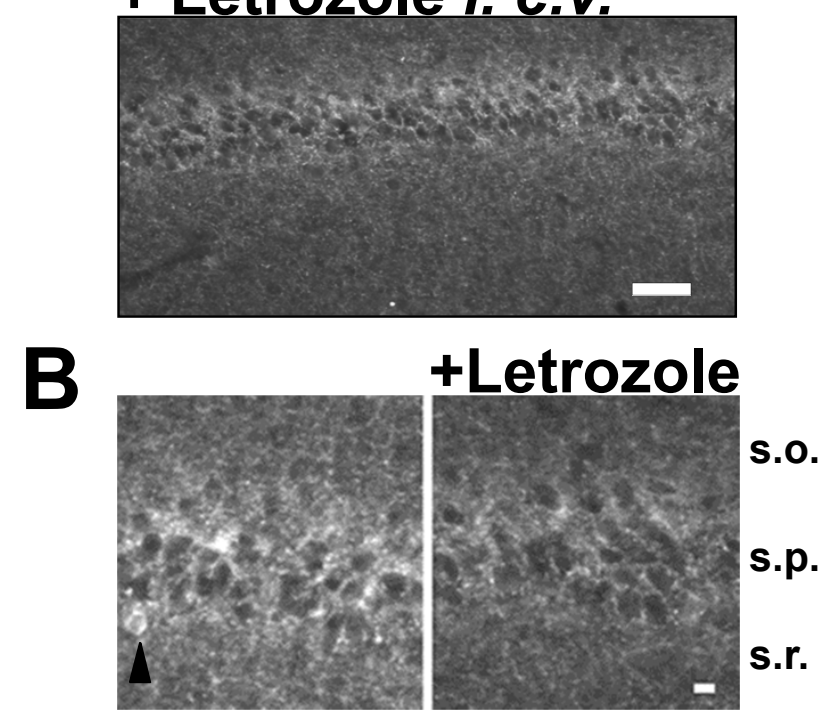

C s. pyramidale

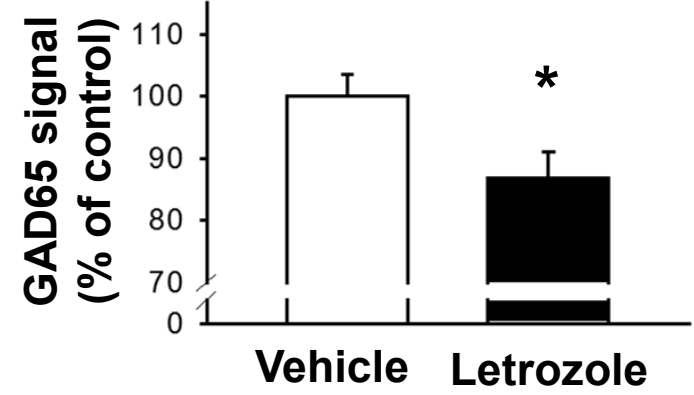


Fig. 5

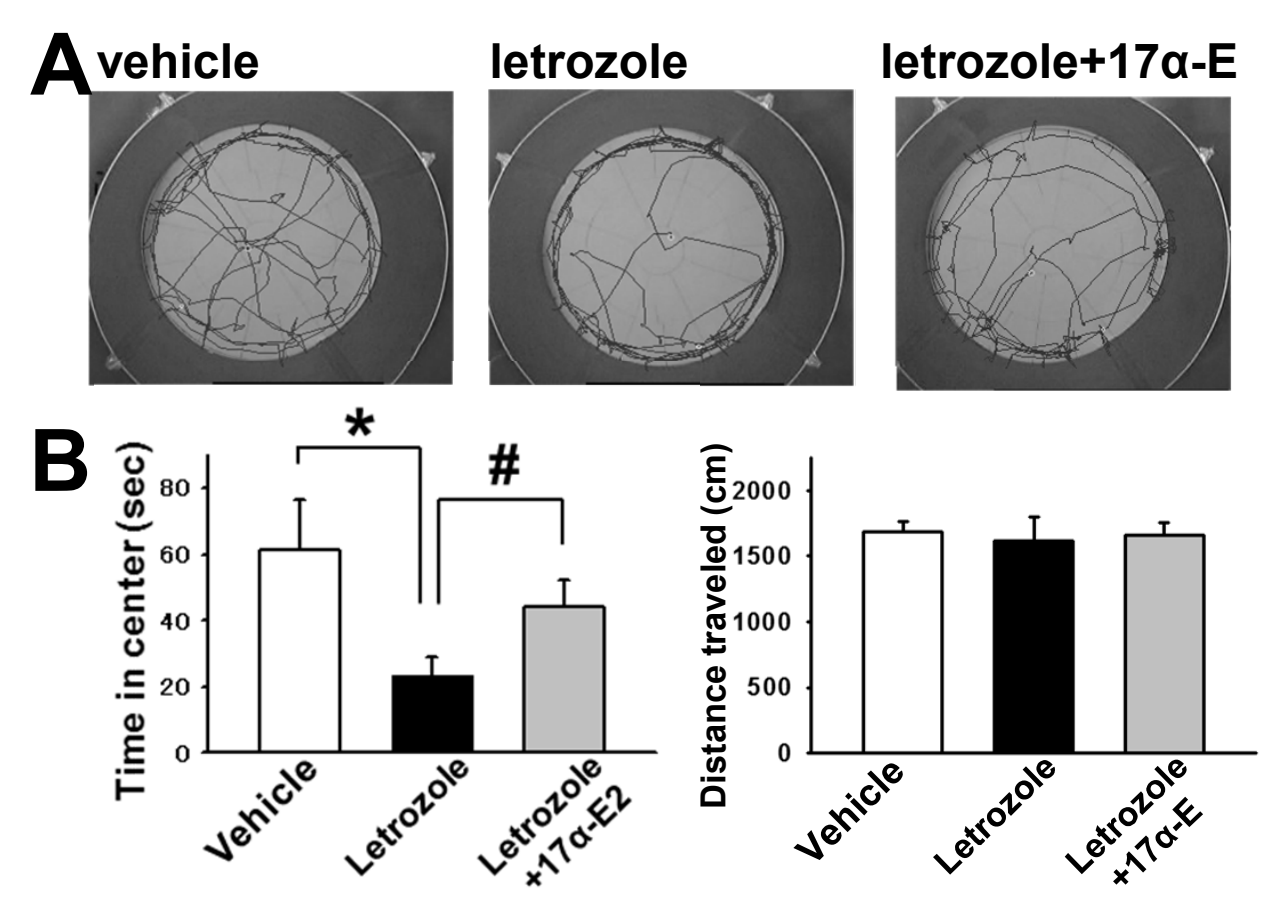

